\section{Surgical management of impacted mandibular premolar}

\author{
Orsola Brucoli, Eleonora Rivetti, Paolo Boffano*
}

CrossMark
Private Practice, Novara, Italy

*Correspondence to: Paolo Boffano, Private Practice, Novara, Italy paolo.boffano@gmail.com

Received: 3 July 2021

Revised: 25 August 2021

Accepted: 30 0ctober 2021

Available Online: 1 December 2021

\title{
Abstract
}

Objective: In this article, we intend to report the management and the outcome of the surgical treatment of an unusual case of impacted mandibular premolar.

Method: A 57-year-old man with an unremarkable medical history was referred by her general dentist for the management of his impacted mandibular left second premolar. The patient complained of an annoying feeling in correspondence of the lingual side of the left mandibular body.

Results: Surgical intervention of the impacted mandibular left second premolar was decided in agreement with the patient under local anesthesia. On the 10th day after intervention, the patient complained no pain, and he showed neither paresthesia nor anesthesia in the region of the chin. No sign of infection was observed. The patient was clinically monitored during the year after the surgery. Six months follow up postoperative panoramic radiograph confirmed a good healing. Conclusion: When surgery is the chosen option, a great attention should be paid not to damage the inferior alveolar nerve. The indication for the removal should be carefully assessed in adult patients.

Keywords: Diagnosis, Impacted, Management, Premolar, Surgery

Cite this Article: Brucoli 0, Rivetti E, Boffano P. 2021. Surgical management of impacted mandibular premolar. Journal of Dentomaxillofacial Science 6(3): 204-206. D0I: 10.15562/jdmfs.v6i3.1273

\section{Introduction}

The impaction of mandibular premolars is a challenging complication in tooth eruption. The arrested eruption of the lower premolars can determine disturbances of mastication and aesthetics. 1-5 Moreover, the frequent tight relationship between the impacted premolar and the inferior alveolar nerve make the management of this condition extremely difficult.

In this article, we report the management and the outcome of the surgical treatment of an unusual case of impacted mandibular premolar.

\section{Case Report}

A 57-year-old man with an unremarkable medical history was referred by her general dentist for the management of his impacted mandibular left second premolar. The patient complained of an annoying feeling in correspondence of the lingual side of the left mandibular body.

Clinical intraoral examination revealed the absence of the left second premolar in the mandibular arch and a hard bulge at the lingual side of the left mandibular body, between the roots of the first premolar and the first molar. A panoramic radiograph confirmed that the left mandibular second premolar was deeply impacted horizontally, with the root in correspondence of the inferior alveolar nerve figure 1 .

Surgical intervention of the impacted mandibular left second premolar was decided in agreement with the patient. Orthodontic traction was not considered to be an option. Surgical intervention was performed under local anesthesia. Local nerve block anesthesia of the inferior dental, lingual, and buccal nerves was induced with 2 capsules of $1.8 \mathrm{~mL}$ of $2 \%$ mepivacaine containing 1:100,000 adrenalin (Carboplyina; Dentsply Italia, Roma, Italy). The left mandibular second premolar was extracted using a lingual full-thickness flap figure 2A. Osteotomy necessary to visualize the impacted premolar was performed using a no. 8 tungsten carbide round bur mounted on a high-speed handpiece figure $2 \mathrm{~B}$. Then, the premolar was luxated figure 3 and removed figures $4 \mathrm{~A}$ and figure $4 \mathrm{~B}$.

Primary closure of the flap was performed using 3- silk suture. The patient was prescribed an antibiotic ( $1 \mathrm{~g}$ of amoxicillin every $12 \mathrm{~h}$ for $5 \mathrm{~d}$ [Zimox; Pfizer Italia, Milan, Italy]), a nonsteroidal anti-inflammatory drug (80 $\mathrm{mg}$ of ketoprofene every $12 \mathrm{~h}$ for $3 \mathrm{~d}$ [OKI granulare; Dompe', L'Aquila, Italy]), corticosteroids (1 mg of betamethasone every $12 \mathrm{~h}$ for $3 \mathrm{~d}$ [Bentelan; Defiante Farmaceutica LDA, Rome, Italy]). Postoperative instructions and use of the prescribed drugs were explained orally and on a printed sheet of paper that was given to the patient. Beginning the day after surgery, patients rinsed twice daily with $0.20 \%$ chlorhexidine solution for 10 days. The sutures were removed 10 days later. On the 10 th day after intervention, the patient complained no pain, and he showed neither paresthesia nor anesthesia in the region of the chin. No sign of infection was observed. The patient was clinically monitored during the year after the surgery. Six 


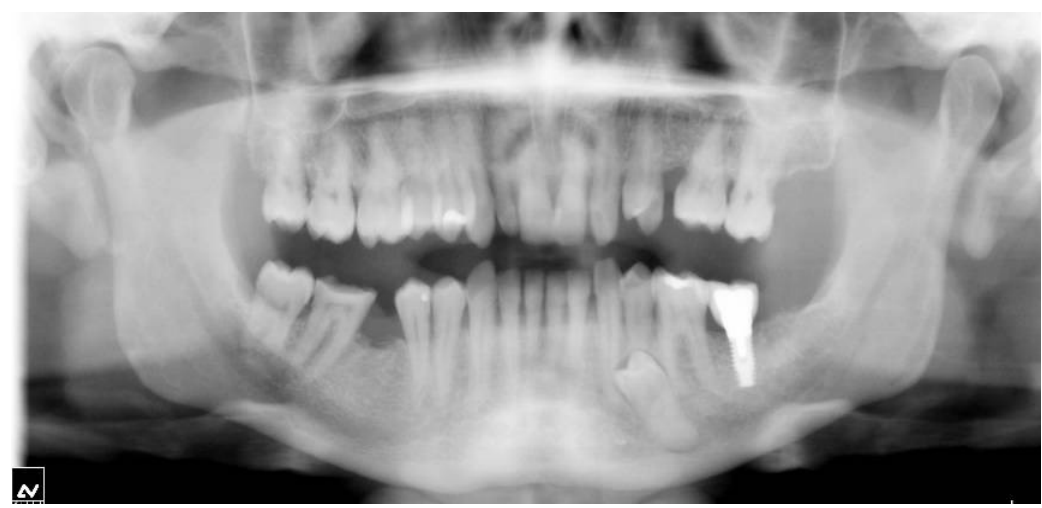

Figure 1 Panoramic radiograph confirming that the left mandibular second premolar was deeply impacted horizontally, with the root in correspondence of the inferior alveolar nerve

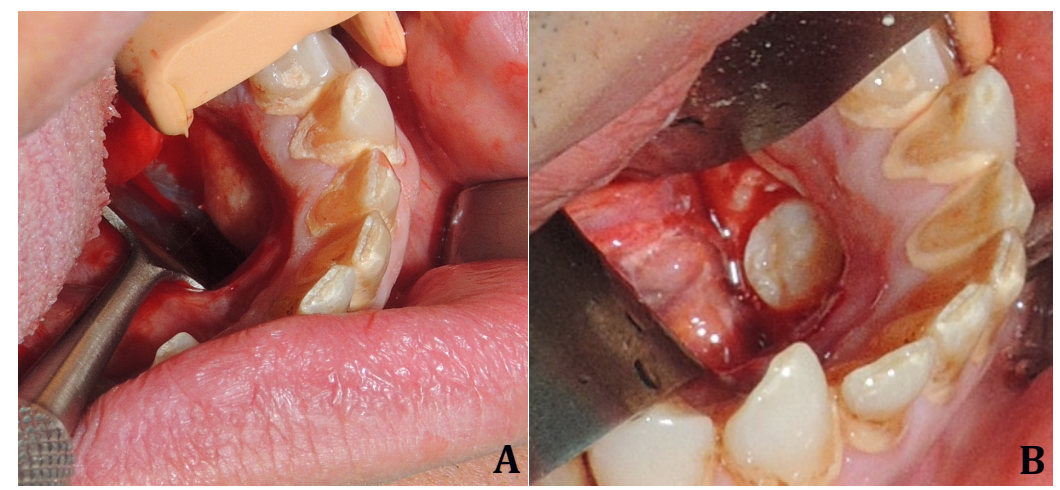

Figure 2 A. Intraoperative view. The left mandibular second premolar was extracted using a lingual full-thickness flap, $B$. Intraoperative view showing the impacted premolar following osteotomy

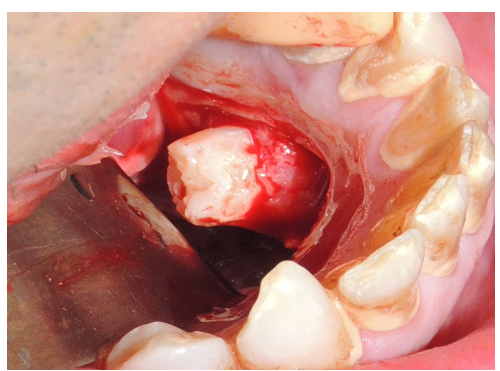

Figure 3 Intraoperative view showing the luxation of the impacted premolar
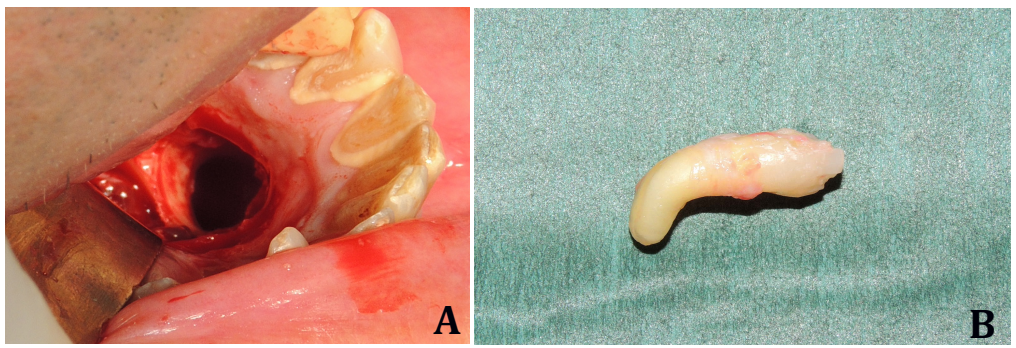

Figure 4 A. Intraoperative view showing the post-extraction socket, B. Image of the removed impacted premolar months follow up postoperative panoramic radiograph confirmed a good healing figure 5.

\section{Discussion}

Removal of impacted teeth is among the most common of procedures performed in oral and maxillo-facial surgery. Even though the procedure is straightforward in the great majority of cases, it can still be plagued by serious complications such as inferior alveolar nerve IAN injury and mandibular fracture..$^{1-5}$ Evidence indicates that depth of impaction, proximity of the tooth to the mandibular canal, surgical technique, intra-operative nerve exposure and the surgeon's experience may be associated with an increased risk of IAN demage.

The management of impacted mandibular premolars in the adult is challenging and in asymptomatic patients, a close observation without surgery is advisable. The surgical removal of impated mandibular premolar can be decided in case of patients complaints or if it is needed because of rehabilitation reasons..$^{1-3}$

Preoperative assessment of surgical difficulty is fundemental to correctly plan the extraction of the impacted premolar.

It is extremely important to assess the various elements that could influence the extraction, such as relative depth, angulation and form of the root, the relationship of the tooth to mandibular canal.

In determining the optimum treatment plan, it might be helpful to tabulate the advantages and disadvantages of each plan. The final choice depends on the weight that we and our patients assign to each factor under consideration. ${ }^{1-5}$

In the present case, we decided to perform a lingual approach to limit the bone removal that was, however, necessary.

Great attention was paid not to damage the inferior alveolar nerve. On the 10th day after intervention, the patient showed neither paresthesia nor anesthesia in the region of the chin. No intraoral dehiscence was present. No signs of infection were observed.

\section{Conclusion}

When surgery is the chosen option, a great attention should be paid not to damage the inferior alveolar nerve. The indication for the removal should be carefully assessed in adult patients. Unfortunately, because of the rarity of this clinical finding, it is difficult to propose clinical procedure protocols. Any single patient should be thoroughly evaluated and management should be individualized. 


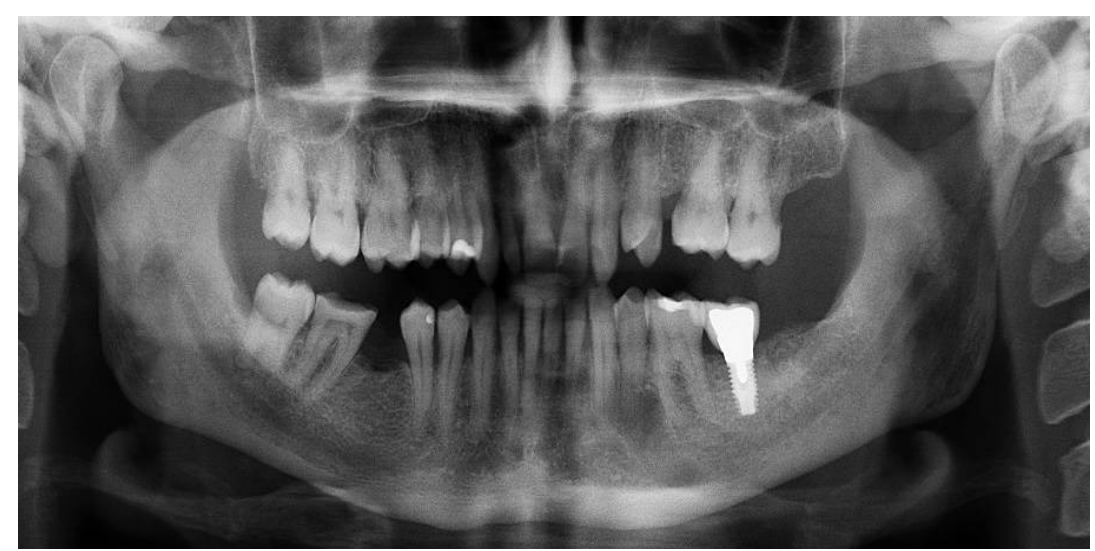

Figure 5 Six months follow up postoperative panoramic radiograph confirming a good healing

\section{Acknowledgment}

The author reports no funding to declare.

\section{Conflict of Interest}

The authors report no conflict of interest.

\section{References}

1. Abdelrahman A, Mahon N, Devahi L, et al. Custom-made drill guides to access impacted mandibular premolars. $\mathrm{Br}$ J Oral Maxillofac Surg 2020;58: 114-115.

2. Halazonetis DJ. Horizontally impacted maxillary premolar and bilateral canine transposition. Am J Orthod Dentofacial Orthop 2009;135: 380-389.

3. Ahuja VR, Jayanna R, Ahuja A, et al. Impacted mandibular premolar concomitant with compound-complex odontome in a 13-year-old Girl: A case report. Int J Clin Pediatr Dent 2020;13: 729-733.

4. Scolozzi P. Buccal corticotomy using piezosurgery as a surgical approach for removal of deeply impacted mandibular teeth: An alternative procedure to avoid pitfalls associated with the conventional technique. J Stomatol Oral Maxillofac Surg 2021;27: S2468-7855.

5. Ishihara Y, Kamioka H, Takano-Yamamoto T, et al. Patient with nonsyndromic bilateral and multiple impacted teeth and dentigerous cysts. Am J Orthod Dentofacial Orthop 2012;141: 228-241.

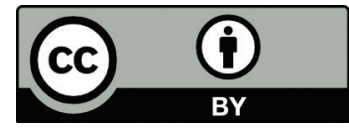

This work is licensed under a Creative Commons Attribution 